\title{
Autopipelining for Data Stream Processing
}

\author{
Yuzhe Tang, Student Member, IEEE, and Buğra Gedik, Member, IEEE
}

\begin{abstract}
Stream processing applications use online analytics to ingest high-rate data sources, process them on-the-fly, and generate live results in a timely manner. The data flow graph representation of these applications facilitates the specification of stream computing tasks with ease, and also lends itself to possible runtime exploitation of parallelization on multicore processors. While the data flow graphs naturally contain a rich set of parallelization opportunities, exploiting them is challenging due to the combinatorial number of possible configurations. Furthermore, the best configuration is dynamic in nature; it can differ across multiple runs of the application, and even during different phases of the same run. In this paper, we propose an autopipelining solution that can take advantage of multicore processors to improve throughput of streaming applications, in an effective and transparent way. The solution is effective in the sense that it provides good utilization of resources by dynamically finding and exploiting sources of pipeline parallelism in streaming applications. It is transparent in the sense that it does not require any hints from the application developers. As a part of our solution, we describe a light-weight runtime profiling scheme to learn resource usage of operators comprising the application, an optimization algorithm to locate best places in the data flow graph to explore additional parallelism, and an adaptive control scheme to find the right level of parallelism. We have implemented our solution in an industrial-strength stream processing system. Our experimental evaluation based on microbenchmarks, synthetic workloads, as well as real-world applications confirms that our design is effective in optimizing the throughput of stream processing applications without requiring any changes to the application code.
\end{abstract}

Index Terms-Stream processing, parallelization, autopipelining

\section{INTRODUCTION}

W ITH the recent explosion in the amount of data available as live feeds, stream computing has found wide application in areas ranging from telecommunications to healthcare to cyber-security. Stream processing applications implement data-in-motion analytics to ingest high-rate data sources, process them on-the-fly, and generate live results in a timely manner. Stream computing middleware provides an execution substrate and runtime system for stream processing applications. In recent years, many such systems have been developed in academia [1], [2], [3], as well as in industry [4], [5], [6].

For the last decade, we have witnessed the proliferation of multicore processors, fueled by diminishing gains in processor performance from increasing operating frequencies. Multicore processors pose a major challenge to software development, as taking advantage of them often requires fundamental changes to how application code is structured. Examples include employing thread-level primitives or relying on higher level abstractions that have been the focus of much research and development [7], [8], [9], [10], [11], [12]. The high-throughput processing requirement of stream processing applications makes them ideal for taking advantage of multicore processors. However, it is a challenge to keep the simple and elegant data flow

- Y. Tang is with the College of Computing, Georgia Institute of Technology, 801 Atlantic Drive, Atlanta, GA 30332. E-mail: yztang@gatech.edu.

- B. Gedik is with the Department of Computer Engineering, Bilkent University, Ankara 06800, Turkey. E-mail: bgedik@cs.bilkent.edu.

Manuscript received 4 Apr. 2012; revised 7 Nov. 2012; accepted 29 Nov. 2012; published online 12 Dec. 2012.

Recommended for acceptance by X.-H. Sun.

For information on obtaining reprints of this article, please send e-mail to: tpds@computer.org, and reference IEEECS Log Number TPDS-2012-04-0349. Digital Object Identifier no. 10.1109/TPDS.2012.333. programming model of stream computing, while best utilizing the multiple cores available in today's processors.

Stream processing applications are represented as data flow graphs, consisting of reusable operators connected to each other via stream connections attached to operator ports. This is a programming model that is declarative at the flow manipulation level and imperative at the flow composition level [13]. The data flow graph representation of stream processing applications contains a rich set of parallelization opportunities. For instance, pipeline parallelism is abundant in stream processing applications. While one operator is processing a tuple, an upstream operator can process the next tuple concurrently. Many data flow graphs contain bushy segments that process the same set of tuples, and which can be executed in parallel. This is an example of task parallelism. It is noteworthy that both forms of parallelism have advantages in terms of preserving the semantics of a parallel program. On the other hand, exploiting data parallelism has additional complexity due to the need for morphing the graph to create multiple copies of an operator and to reestablish the order between tuples. Pipeline and task parallelism do not require morphing the graph and preserve the order without additional effort. These two forms of parallelism can be exploited by inserting the right number of threads into the data flow graph at the right locations. It is desirable to perform this kind of parallelization in a transparent manner, such that the applications are developed without explicit knowledge of the amount of parallelism available on the platform. We call this process autopipelining.

There are several challenges to performing effective and transparent autopipelining in the context of stream processing applications.

First, optimizing the parallelization of stream processing applications requires determining the relative costs of 
operators. The prevalence of user-defined operators in realworld streaming applications [5] means that cost modeling, commonly applied in database systems [14], is not applicable in this setting. On the other hand, profile-driven optimization that requires one or more profile runs based on compilergenerated instrumentation [15], [16], while effective, suffers from usability problems and lack of runtime adaptation. On the usability side, requiring profile runs and specification of additional compilation options has proven to be unpopular among users in our own experience (see Appendix J, which can be found on the Computer Society Digital Library at http://doi.ieeecomputersociety.org/10.1109/TPDS. 2012.333). In terms of runtime adaptation, the profile run may not be representative of the final execution. In summary, a light-weight dynamic profiling of operators is needed to provide effective and transparent autopipelining.

Second, and more fundamentally, it is a challenge to efficiently (time-wise) find an effective (throughput-wise) configuration that best utilizes available resources and harnesses the inherent parallelism present in the streaming application. Given $N$ operator ports and up to $T$ threads, there are combinatorial possibilities, $\sum_{k=0}^{T}\left(\begin{array}{c}N \\ k\end{array}\right)$ to be precise. In the absence of autopipelining, we have observed application developers struggling to insert threads manually $^{1}$ to improve throughput. This is no surprise, as for a medium size application with 50 operators on an 8-core system, the number of possibilities reach multiple billions. Thus, a practical optimization solution needs to quickly and automatically locate an effective configuration at runtime.

Finally, deciding the right level of parallelism is a challenge. The behavior of the system is difficult to predict for various reasons. User-defined operators can contain locks that inhibit effective parallelization. The overhead imposed by adding an additional thread in the execution path is a function of the size of the tuples flowing through the port. The behavior of the operating system scheduler cannot be easily modeled and predicted. The impact of these and other system artifacts are observable only at runtime and treated as a blackbox. While the optimization step can come up with threading configuration changes that are expected to improve performance, such decisions need to be tried out and dynamically evaluated to verify their effectiveness. As such, we need a control algorithm that can backtrack from bad decisions.

In this paper, we describe an autopipelining solution that addresses all of these challenges. It consists of:

- A light-weight runtime profiling scheme that uses a novel metric called per-port utilization to determine the amount of time each thread spends downstream of a given operator input port.

- A greedy optimization algorithm that finds locations in the data flow graph where inserting additional threads helps eliminate bottlenecks and improve throughput.

- A control algorithm that decides when to stop inserting additional threads and also backtracks from decisions that turn out to be ineffective.

1. SPL language [5] provides a configuration called "threaded port" that can be used to manually insert threads into a data flow graph.

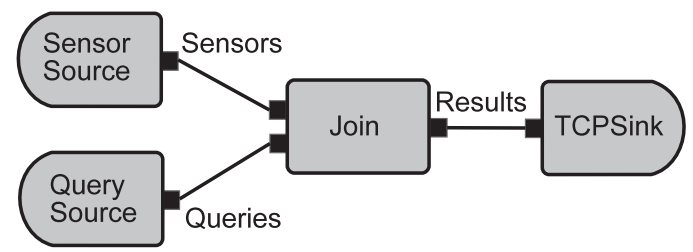

Fig. 1. Data flow graph for the SensorQuery app.

- Runtime mechanics to insert/remove threads while maintaining lock correctness and continuous operation.

We implemented our autopipelining solution on IBM's system S [3] -an industrial strength stream processing middleware. We evaluate its effectiveness using microbenchmarks, synthetic workloads, and real-world applications. Our results show that autopipelining provides better throughput compared to hand-optimized applications at no cost to application developers.

\section{BACKGROUND}

We provide a brief overview of the basic concepts associated with stream processing applications, using SPL [5] as the language of illustration. We also describe the fundamentals of runtime execution in System S.

\subsection{Basic Concepts}

Listing 1 in Appendix A, which is available in the online supplemental material, gives the source code for a very simple stream processing application in SPL, with its visual representation depicted in Fig. 1 below.

The application is composed of operator instances connected to each other via stream connections. An operator instance is a vertex in the application graph. An operator instance is always associated with an operator. For instance, the operator instance shown in the middle of the graph in Fig. 1 is an instance of a Join operator. In general, operators can have many different instantiations, each using different stream types, parameters, or other configurations such as windows. Operator instances can have zero or more input and output ports. Each output port generates a uniquely named stream, which is a sequence of tuples. Connecting an output port to the input of an operator establishes a stream connection. Operators are often implemented in general purpose languages, using an event driven interface, by reacting to tuples arriving on operator input ports. Tuple processing generally involves updating some operator-local state and producing result tuples that are sent out on the output ports.

There are two important aspects of real-world applications that are highly relevant for our work:

- Real-world applications are usually much larger in terms of the number of operators they contain, reaching hundreds or even thousands.

- Real-world applications contain many user-defined re-usable operators to implement cross-domain or domain-specific manipulations.

The former point motivates the need for automatic parallelization, whereas the latter motivates the need for dynamic profiling. 


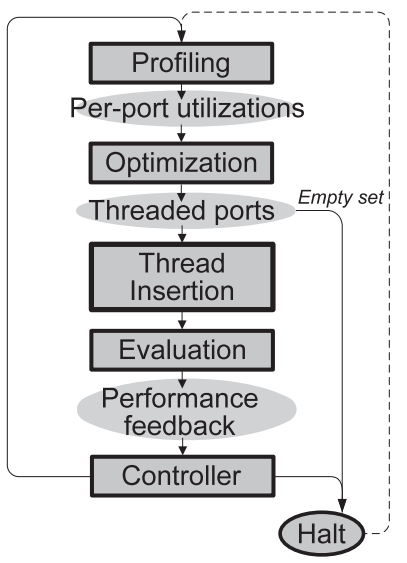

Fig. 2. Overview of the autopipelining system.

\subsection{Execution Model}

A distributed stream processing middleware, such as System S, executes data flow graphs by partitioning them into basic units called processing elements. Each processing element contains a subgraph and can run on a different host. For small- and medium-scale applications, the entire graph can map to a single processing element. Without loss of generality, in this paper, we focus on a single multicore host executing the entire graph. Our autopipelining technique can be applied independently on each host when the whole application consists of multiple, distributed processing elements.

There are two main sources of threading in our streaming runtime system, which contribute to the execution of the data flow graphs. The first one is operator threads. Source operators, which do not have any input ports, are driven by a separate thread. When a source operator makes a submit call to send a tuple to its output port, this same thread executes the rest of the downstream operators in the data flow graph. As a result, the same thread can traverse a number of operators, before eventually coming back to the source operator to execute the next iteration of its event loop. This behavior is because the stream connections in a processing element are implemented via function calls. Using function calls yields fast execution, avoiding scheduler context switches, and explicit buffers between operators. We refer to this optimization as operator fusion [16], [15]. Nonsource operators can also create operator threads, but this is rare. In general, the number and location of operator threads are not flexible because they are dictated by the application and the operator implementations.

The second source of threading is threaded ports. Threaded ports can be inserted at any operator input port. When a tuple reaches a threaded port, the currently executing thread will insert the tuple into the threaded port buffer, and go back to executing upstream logic. A separate thread, dedicated to the threaded port, will pick up the queued tuples and execute the downstream operators. Threaded port buffers are implemented as cache-optimized concurrent lock-free queues [17].

The goal of our autopipelining solution is to automatically place threaded ports at operator input ports during runtime, so as to maximize throughput.

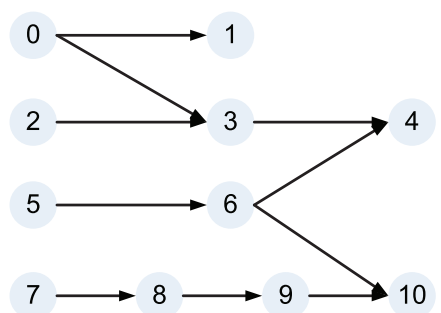

Fig. 3. Operator graph.

\section{System Overview}

In this section, we give an overview of our autopipelining solution. Fig. 2 depicts the functional components and the overall control flow of the solution. It consists of five main stages that run in a continuous loop until a termination condition is reached.

The first stage is the profiling stage. In this stage a lightweight profiler determines how much time each of the existing threads spend on executing the operators in the graph. This profiling information, termed per-port utilization, is used as input to the optimization stage. An optimization algorithm that uses a greedy heuristic determines what the next action should be. The next action could either be to halt, as it could find nothing but an empty set of threaded ports at this time, or it could be to add additional threads at specific input ports. If the optimizer decides to add new threads, then the thread insertion component applies this decision. This is followed by the evaluation component, which evaluates the performance of the system after the thread insertions. The performance results from the evaluation are put into the controller component as a feedback, which takes one of two possible actions. It could vet all the thread insertions and go to the next iteration of the process. Alternatively, it could remove some or all of the inserted threads, reverting back the decisions taken by the optimizer. This could be followed by moving to the next iteration of the process or halting the process. In the former case, it applies a blacklisting algorithm to avoid coming up with the same ineffective configuration in the next iteration.

The system can be taken out of the halting state in case a shift in the workload conditions is detected. However, the focus of this work is on finding an effective operating point right after the application launch.

\subsection{An Example Scenario}

Throughout the paper we use an example application to illustrate various components of our solution. The compiletime and runtime data flow graphs for this application are given in Figs. 3 and 4, respectively. For simplicity of exposition, we assume that all operators have a single input port and a single output port. However, our solution trivially extends to the general case and has been implemented and evaluated for the multiport scenario (see Section 8).

The sample application consists of an 11-operator graph as shown in Fig. 3. There are four source operators (namely, $o_{0}, o_{2}, o_{5}$, and $o_{7}$ ) which generate tuples. At runtime, there are four threads initially, $t_{0}, \ldots, t_{3}$, that execute the program, assuming no threaded ports have been inserted. 


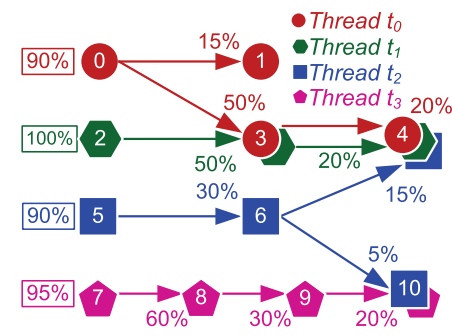

Fig. 4. Runtime op. graph.

Fig. 4 shows the execution path of different threads in different colors and shapes. Note that some operators are present in the execution path of multiple threads. For instance, threads $t_{0}$ and $t_{1}$ share operator $o_{3}$ in their execution paths.

The runtime graph cannot be derived solely from the compile-time graph. The paths threads take can depend on tuple runtime values, as well as operator runtime behavior, such as selectivity or tuple submission decisions. Hence, the compile-time graph restricts each thread in terms of what operators it can traverse, but does not exactly define its path. We derive the runtime graph based on runtime profiling (see Section 6).

We now look at the metrics that will help us formulate the autopipelining problem.

\subsubsection{Profiling Metrics}

The main profiling metric collected by our autopipelining solution is called the per-port utilization, which we denote with $\mu(o, t)$. The variable $o$ represents any arbitrary operator, and $t$ represents any thread that can execute that operator. We define the utilization, $\mu(o, t)$, to be the amount of CPU utilized by thread $t$ when executing all downstream operators starting from the input port of operator $o$. During program execution, the profiler maintains $\mu(o, t)$ for every operator/thread pair for which the thread $t$ executes the operator $o$. In Fig. 4, for example, the input port of operator $o_{6}$ is associated with utilization 30 percent, meaning that thread $t_{2}$ spends 30 percent of the CPU time on executing $O_{6}$ and its downstream operators, which are operators $o_{4}$ and $o_{10}$. Thus, $\mu\left(o_{6}, t_{2}\right)=0.3$.

For each thread, we also define per-thread utilization, denoted as $\mu(t)$, which is the overall CPU utilization of thread $t$. For example, in Fig. 4 , thread $t_{2}$ has a utilization of 90 percent, thus $\mu\left(t_{2}\right)=0.9$.

The reason we pick per-port CPU utilization, $\mu(o, t)$, as our main profiling metric is that it simplifies predicting the relative work distribution between threads after inserting a new thread on an input port. For instance, if a threaded port is being added in front of operator $o_{6}$ in Fig. 4, the newly created thread will take 30 percent CPU utilization from the existing thread $t_{2}$.

Predicting the relative work distribution for a potential thread insertion is performed in the following way. Assume that $T(o)=\{t \mid \mu(o, t)>0\}$ denotes the list of threads that contain a given operator $o$ in their execution path. Adding a threaded port at operator $o$ will have two consequences. First, all of the threads in $T(o)$ will execute only up to the input port of operator $o$. Second, a new thread, $t^{\prime}$, will execute the rest of the executions paths for all threads in
$T(o)$. The prediction of the work distribution for the newly created thread $t^{\prime}$ is $\mu^{\prime}\left(t^{\prime}\right)=\sum_{t \in T(o)} \mu(o, t)$. For an existing thread $t \in T(o)$, the prediction is $\mu^{\prime}(t)=\mu(t)-\mu(o, t)$. For instance, in Fig. 4, when a threaded port is added to operator $o_{3}$, we predict $\mu^{\prime}\left(t_{0}\right)=0.4, \mu^{\prime}\left(t_{1}\right)=0.5$, and $\mu^{\prime}\left(t^{\prime}\right)=1$.

It is important to note that $\mu^{\prime}$ is a relative metric of how the work is partitioned between the existing threads and the newly created thread. It is not an accurate prediction of what the CPU utilizations will be after the thread insertion. The expectation is that, given enough processing resources and enough work present in the application, the actual utilizations $(\mu)$ will be higher than the relative predictions $\left(\mu^{\prime}\right)$. For instance, consider a simple chain of operators executed by a single thread that has $\mu\left(t_{0}\right)=1$. Adding a threaded port in the middle of this chain will result in $\mu^{\prime}\left(t_{0}\right)=0.5$ and $\mu^{\prime}\left(t_{1}\right)=0.5$. We use these relative utilization values to assess whether or not inserting a new thread in this location will improve performance. After the insertion, the optimistic expectation is that $\mu\left(t_{0}\right)=\mu\left(t_{1}\right)>$ 0.5 , because $u^{\prime}\left(t_{0}\right)<1$ and $u^{\prime}\left(t_{1}\right)<1$, which leaves room for improvement in throughput. The evaluation and control stages of our solution deal with cases where this expectation does not hold.

\subsubsection{Utility Function}

The predicted relative utilizations are used to define a utility function that measures a threaded port insertion's goodness. Given an insertion at operator $o$, causing the creation of thread $t^{\prime}$, we define its utility as

$$
U\left(o, t^{\prime}\right)=\max \left(\mu^{\prime}(t) \mid t \in T(o) \cup\left\{t^{\prime}\right\}\right) .
$$

The utility function for a given operator and its new thread is the largest predicted relative work distribution across all of the threads with that operator in its path. Our goal is to minimize this utility function. The intuition behind the utility function is simple: the thread that has the highest predicted work $\left(\mu^{\prime}\right)$ will become the bottleneck of the system.

Suppose $T(o)=\left\{t_{0}\right\}$ and our predictions after insertion of a new thread $t^{\prime}$ at operator $o$ are $\mu^{\prime}\left(t_{0}\right)=0.3$ and $\mu^{\prime}\left(t^{\prime}\right)=0.6$. The utility of this insertion is $U\left(o, t^{\prime}\right)=\max (0.6$, $0.3)=0.6$. A better insertion at a different operator $o^{\prime}$, where $T\left(o^{\prime}\right)=\left\{t_{0}\right\}$, that would give a lower utility value is: $\mu^{\prime}\left(t_{0}\right)=0.5$ and $\mu^{\prime}\left(t^{\prime}\right)=0.5$, leading to $U\left(o^{\prime}, t^{\prime}\right)=0.5$. However, it may not always be possible to find such an insertion based on the per-port utilizations of the operators reported by profiling.

For a set of thread insertions, say $C=\left\{\left\langle o, t^{\prime}\right\rangle\right\}$, we define an aggregate utility function $U(C)$ as

$$
U(C)=\max \left(U\left(o, t^{\prime}\right) \mid\left\langle o, t^{\prime}\right\rangle \in C\right) .
$$

Here, we pick the maximum of the individual utilities. We will further discuss and illustrate the aggregate utility function shortly.

\subsection{The Optimization Problem}

Recall that the goal of the optimization stage is to find one or more threaded ports that will improve the throughput of 
the system. We propose the following heuristic for the optimization stage:

Minimize the aggregate utility function while making sure that one and only one threaded port is inserted in the execution path of each heavily utilized thread.

This formulation is based on three core principles:

1. Help the needy. At each step, we only insert threads in the execution path of heavily utilized threads. A heavily utilized thread is a bottleneck, which implies that if it has more resources, overall throughput will improve.

2. Be greedy but generous. By definition our solution is greedy, as at each step it comes up with incremental insertions that will improve performance. However, inserting a single thread at a time does not work, which is why we make sure that a thread is inserted in the execution path of each heavily utilized thread. To see this point, consider the scenario where two threads execute a simple chain of four equal sized operators. The first thread executes the first two operators, and the second thread executes the remaining two. As an incremental step, if we only help the first thread, we will end up having three threads, where the last thread still executes two operators. This imbalance will become the bottleneck and, thus, the throughput will not increase. But, if we help both of the two original threads, we expect the throughput to increase.

A more subtle, but critical, point is the requirement that one and only one thread is added to the execution path of each heavily utilized thread. This is strongly related to the greedy nature of the algorithm. If we are to insert more than one thread in the execution path of a given thread, then the prediction of a thread's $\mu^{\prime}$ requires significantly more profiling information (such as the amount of CPU time a thread spends downstream of a port when it reaches that port by passing through a given set of upstream input ports). We want to maintain a light-weight profiling stage that will not disturb application performance during profiling. Hence, we make our algorithm greedy by inserting at most one thread in the execution path of an existing thread, but for each one of the heavily utilized threads (thus generous).

3. Be fair. We minimize the utility function $U$, which means that new threads are inserted such that the newly created and the existing threads have balanced load.

\section{Optimization Algorithm}

We now describe a base optimization algorithm and a set of enhancements that improve its running time. A cost analysis is provided in Appendix B, which is available in the online supplemental material.

\subsection{The Algorithm}

For a simple chain of operators, designing an algorithm that meets the criteria given in Section 3.2 is straightforward.
However, operators that are shared across threads complicate the design in the general case. We need to make sure that one and only one thread is inserted in the execution path of each existing thread, even though the same thread can be inserted in the execution path of multiple existing threads. The main idea behind the algorithm is to reduce the search space via selection and removal of shared operators from the set of possible solutions, and then explore each subspace separately.

Before describing the algorithm in detail, we first introduce a simple matrix form that represents a subspace of possible solutions.

Matrix representation. For each thread, we initially have all the operators in the execution path of it as a possible choice for inserting a threaded port. As the algorithm progresses, we gradually remove some of the operators from the list to reduce the search space. For instance, the runtime operator graph from Fig. 4 can be converted into the following matrix representation:

$$
\begin{gathered}
t_{0} \\
t_{1} \\
t_{2} \\
t_{3}
\end{gathered}\left(\begin{array}{cccc}
o_{0}, 90 \% & o_{1}, 15 \% & o_{3}, 50 \% & o_{4}, 20 \% \\
o_{2}, 100 \% & o_{3}, 50 \% & o_{4}, 20 \% & \\
o_{5}, 90 \% & o_{6}, 30 \% & o_{4}, 15 \% & o_{10}, 5 \% \\
o_{7}, 95 \% & o_{8}, 60 \% & o_{9}, 30 \% & o_{10}, 20 \%
\end{array}\right)
$$

The matrix contains one row for each thread in the unmodified application. For each row, it lists the set of operators that is in the execution path of the thread with their associated CPU utilization metrics, which is $\mu(o, t)$. Note that the source operators are placed on the first column and are separated from the rest. They are not considered as potential places to add threaded ports as they have no input ports. We exclude them from the matrix representation for the remainder of the paper. The remaining operators are in no particular order, but we sort them by their index for ease of exposition.

The algorithm is composed of four major phases, namely, bottleneck selection, solution reduction, candidate formation, and solution selection.

Bottleneck selection. The first phase is the bottleneck selection, which identifies highly utilized threads. A threshold $\beta \in[0,1]$ is used to eliminate threads whose CPU utilizations are below it. For instance, if $\beta=0.92$, threads $t_{0}$ and $t_{2}$ are eliminated since their utilizations are smaller than the threshold and, thus, are not deemed bottlenecks. For the rest of this section, we assume $\beta=0.8$ for the running example, which means all of the four threads are considered as bottlenecks.

Solution reduction. The second phase is the solution reduction, which performs a tree search to reduce the solution space. At the root of the tree is the initial matrix. At each step, we choose one of the leaf matrices that still contains shared operators based on the runtime data flow graph. We pick one of these shared operators for that leaf matrix and perform selection and removal to yield two submatrices in the tree.

Selection means that we select the shared operator as a part of the solution, and thus remove all other operators from the rows that contain the shared operator. Furthermore, we remove all operators that originally appeared together with the shared operator in the same row, from other rows, since 


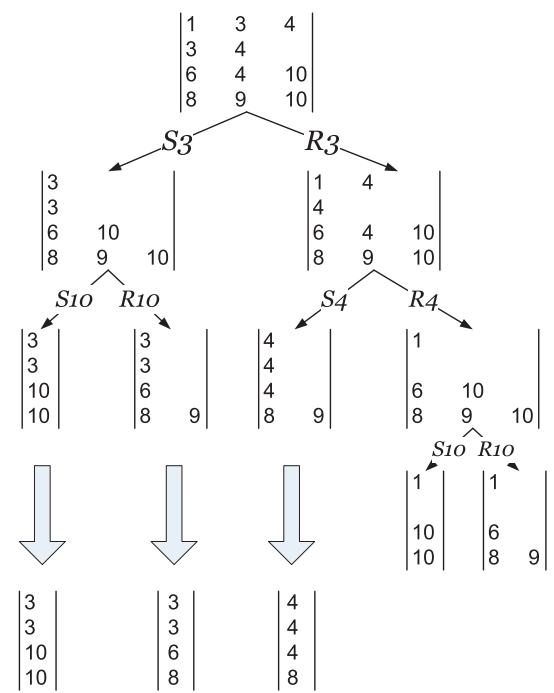

Fig. 5. Solution reduction and candidate formation.

they cannot be selected in a valid solution. Fig. 5 shows an example. Consider the edge labeled $S 3$, which represents the case of selecting shared operator 3 . After the selection, the first two rows now have operator 3 as the only choice. Furthermore, operators 1 and 4-which previously appeared in the same row as 3-are removed from all rows, as picking them would result in inserting more than one thread on the execution paths of the first two threads.

Removal means we exclude the shared operator from the solution, and thus we remove it from all rows where it appears. Fig. 5 shows an example. Consider the edge labeled $R 3$, which represents the case of removing the shared operator 3 .

The solution reduction phase continues until the leaf matrices have all of their shared operators removed.

Candidate formation. After the solution reduction phase, all leaves of the tree contain precandidate solutions. The goal of the candidate formation phase is to create candidate solutions out of the precandidate ones. As a part of candidate formation, first we apply a filtering step. If we encounter a leaf matrix where a thread is left without an operator in its row, yet there is another dependent thread that has a nonempty row, then we eliminate this leaf matrix. We consider threads that share operators in their execution paths as dependent.

As an example, the rightmost two leaves in Fig. 5 are removed in the filtering step. After the filtering step, we convert each remaining precandidate solution into a candidate solution by making sure that each nonempty row contains a single operator, i.e., we convert each matrix into a column vector. When there are multiple operators in a row, we compute the utility function $U(o, t)$ for each, and pick the one that gives the lowest value. As an example, the precandidate solution pointed at by arrow $S 4$ in Fig. 5 is converted into a candidate solution by picking operator 8 as opposed to operator 9 . Operator 8 has a lower utility value, $U\left(o_{8}, t_{3}\right)=0.6$, compared to operator 9's utility, $U\left(o_{9}, t_{3}\right)=0.65$.

Solution selection. In the solution selection phase, we pick the best candidate among the ones produced by the candidate formation phase. Recall that our utility function $U(o, t)$ was defined on a per-thread basis. To pick the best candidate, we use the aggregate utility function $U(C)$, where $C=\{\langle o, t\rangle\}$ represents a candidate solution. Recall that we pick the maximum of the individual utilities, ${ }^{2}$ thus $U(C)=\max (U(o, t) \mid\langle o, t\rangle \in C)$. We pick the candidate solution with the minimum aggregate utility as the final solution. In the running example, this corresponds to picking $C=\left\{\left\langle o_{4}, t_{0}\right\rangle,\left\langle o_{4}, t_{1}\right\rangle,\left\langle o_{4}, t_{2}\right\rangle,\left\langle o_{8}, t_{3}\right\rangle\right\}$ with aggregate utility of 0.8 .

\subsection{Algorithm Enhancements}

We further propose and employ two enhancements to our basic algorithm.

Pruning. Our enhanced algorithm stops branching when it finds that the utility function value for some of the rows in the current matrix is already equal to or larger than 100 percent. For example, in Fig. 5, there is no point to continue branching after $R 4$, since thread $t_{1}$ has no potential threaded port to add and will remain bottlenecked after inserting other threads.

Sorting. In the solution reduction phase, we use the degree of operator sharing as our guideline for picking the next solution to further reduce. We sort the shared operators based on the number of rows they appear in. This way, if a shared operator shows up in the execution path of many threads, it is considered earlier in the exploration as it will result in more effective reduction in the search space, especially when used with pruning. When selected, shared operators have a higher chance of causing the utility function value to go over 100 percent due to contribution from multiple threads.

\section{Evaluation and Control}

The thread insertions proposed by the optimization stage are put into effect by the runtime. After inserting the new threads, the evaluation stage measures the throughput on input ports which received a threaded port. The throughput is defined as the number of tuples processed per second. If the throughput increased for all of the input ports that has received a threaded port, then the controller stage moves on to the next iteration.

If the throughput has not increased for some of the input ports, then the control stage performs blacklisting. The ports for which the throughput has not improved are blacklisted. Furthermore, the thread insertions are reverted by removing these threaded ports from the flow graph. Blacklisted input ports are excluded from consideration in future optimization stages. If the percentage of blacklisted input ports exceeds a predefined threshold $\alpha \in[0,1]$, then the process halts. Otherwise, we move on to the next iteration. It is possible that the process halts even before the threshold $\alpha$ is reached, as a feasible solution may not be found during the optimization stage.

Alternative blacklisting policies can be applied to reduce the change of getting stuck at a local minima. For instance, the blacklisted ports can be maintained on a per-pipelining

2. When there are more than one dependent thread groups, utility $U$ is computed independently for each group and the maximum is taken as the final aggregate utility. 
configuration basis rather than globally, at the cost of keeping more state around.

\section{Profiler}

We describe the basic design of the profiler component. The implementation details can be found in Appendix C, which is available in the online supplemental material.

Our profiler follows the design principle of gprof [18], that is, to use both instrumentation and periodic sampling for profiling. However, the instrumentation is not a part of the generated code. Instead, the SPL runtime has lightweight instrumentation which records thread activity with respect to operator execution. More specifically, the instrumented SPL runtime monitors the point at which a thread enters or exits an input port, so that it can track which ports are currently active. It uses a special per-thread stack, called the E-stack, for this purpose.

To collect the amount of CPU time a thread spends downstream of an input port, our system periodically samples the thread status and traverses the E-stacks. We call the period between two consecutive samplings the sampling period, denoted by $p_{s}$. If there are $N$ occurrences during the last $p_{o}$ seconds where thread $t$ was found to be active doing work downstream of operator $o$ 's input port, then the per-port thread utilization $\mu(o, t)$ is given by $\frac{N}{p_{o} / p_{s}}$. The intuition for this calculation is that it is the number of observations $(N)$ divided by how many times we sample during a given time period $\left(p_{o} / p_{s}\right)$.

Periodic sampling is inherently subject to statistical inaccuracy, thus enough samples should be collected for accurate results. This could be achieved by either increasing the duration of profiling $\left(p_{o}\right)$ or decreasing the sampling period $\left(p_{s}\right)$. Given the long running nature of streaming applications, we favor the former approach.

\section{Dynamic Thread InSERTION/REMOVAL}

Thread insertion and removal is implemented by dynamically adding and removing threaded ports. Both activities require suspending the current flow of data for a very brief amount of time, during which the circular buffer associated with the threaded port is added/removed to/from the data flow graph. Finally, the suspended flow is resumed. Suspending the flow, however, is not the only step necessary to preserve safety. In the presence of stateful operators, dynamic lock insertion and removal is required to ensure mutually exclusive access to shared state. This is further discussed in Appendix D, which is available in the online supplemental material. Our implementation does make use of thread pools, since the additional work that is performed during thread injection and removal dominates the overall cost.

\section{Experimental Results}

We evaluate the effectiveness of our solution based on experimental results. We perform three kinds of experiments. First, we use microbenchmarks to evaluate the components of our solution and verify the assumptions that underlie our techniques. Second, we evaluate the running

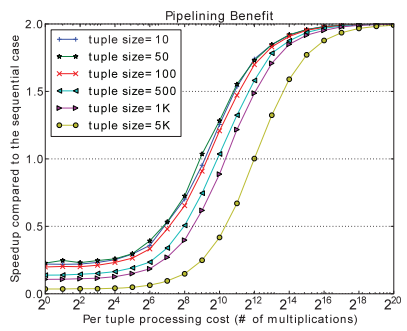

Fig. 6. Speedup versus processing cost.

time efficiency of our optimization algorithm under varying topologies and application sizes, using synthetic applications. Third, using three real-world applications, we compare the throughput our autopipelining scheme achieves to that of manual optimization as well as no optimization. The second set of experiments, based on synthetic applications, can be found in Appendix F, which is available in the online supplemental material.

\subsection{Experimental Setup}

We have implemented our autopipelining scheme in $\mathrm{C}++$, as a part of the SPL runtime within System S [3].

All of our experiments were performed on a host with 2 Intel Xeon processors. Each processor has four cores, and each core is a two-way SMT, exposing 16 hardware threads per node, but only eight independent cores. When running the experiments, we turn off hyperthreading so that the number of virtual cores equals the number of physical cores (which is 8). ${ }^{3}$

\subsection{Microbenchmarks}

For the microbenchmarks, we use a simple application topology that consists of a chain of eight operators. All operators have the same cost and perform the same operation (a series of multiplications). The cost of an operator is configurable. Plots for cost-throughput tradeoff are given in Appendix E, which is available in the online supplemental material.

\subsubsection{Pipelining Benefit}

Pipelining is beneficial under two conditions. First, enough hardware resources should exist to take advantage of an additional thread. Second, the overhead of copying a tuple to a buffer and a thread switch-over should be small enough to benefit from the additional parallelism. When these conditions do not hold, the evaluation and control stages of our auto-pipelining solution will detect this and adjust the adaptation process.

We evaluate the pipelining benefit and show how it relates to the overhead associated with threaded ports by measuring the speedup obtained when executing our application with two threads instead of one. Fig. 6 plots the speedup as a function of the per-tuple processing cost, for different tuple sizes. When the per-tuple processing cost is small, it is expected that using an additional thread will introduce significant overhead. In fact, we observe that the additional thread reduces the performance (speedup less than 1). As the per-tuple processing cost gets higher, we see that perfect speedup of $2 \times$ is achieved. The tuple sizes also

3. This is done to avoid impacting the scalability micro-benchmarks. 


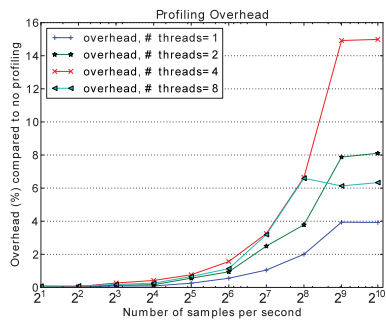

Fig. 7. Profiling overhead versus sampling rate.

have an impact on the benefit of pipelining. For large tuple sizes, the additional copying required to go through a buffer creates overhead. Thus, the crossover point for achieving $>1 \times$ speedup happens at a lower per-tuple cost for smaller sized tuples. For small tuples, custom allocators [19] can be used to further improve the performance. For large tuples, the copying of the data contents dominates the cost. While copy-on-write (COW) techniques can be used to avoid this cost, it is well accepted that COW optimizations are not effective in the presence of multithreading.

\subsubsection{Profiling Overhead}

Light-weight profiling that does not disturb application performance is essential for performing autopipelining at runtime. In Fig. 7, we study the profiling overhead. The overhead is defined as the percent reduction in the throughput compared to the nonprofiling case. The figure plots the overhead as a function of the number of samples taken per second, for different number of threads. The operators are evenly distributed across threads. We observe that, as a general trend, the profiling overhead increases as the profiling rate grows. For the remainder of the experiments in this paper, we use a profiling sampling rate of $p_{s}=100$, which corresponds to a 3 percent reduction in performance. Note that the profiler is only run for a specific period (for $p_{o}$ seconds) during one iteration of the adaptation phase. Once the adaptation is complete, no overhead is incurred due to profiling.

We further observe that increasing the profiling rate beyond a threshold does not increase the overhead anymore. This is because the system starts to skip profiling signals when the sampling period $p_{s}$ is shorter than the time needed to run the logic associated with the profiling signal. Interestingly, the profiling overhead does not monotonically increase with the number of threads. At first glimpse, this may be unexpected since more threads means more execution stacks to go through during profiling. However, with more threads, each execution stack has less entries, which decreases the overhead.

For most operator graphs, it is the depth of the operator graph that impacts the worst case profiling cost, rather than the number of threads used. For instance, for a linear chain, the number of stack entries to be scanned only depends on the depth of the graph. For bushy graphs this number can also depend on the number of threads, even though it is rarely linear in the number of threads in practice (a reverse tree is the worst case).

\subsubsection{Impact of Threads}

Recall that one of the principles of our optimization is to insert a threaded port in the execution path of each

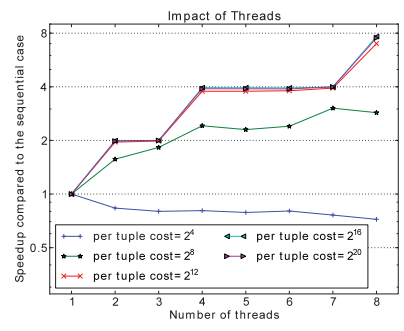

Fig. 8. Speedup for different \# of threads.

bottleneck thread. We do this because the speedup from adding threads one-at-a-time will result in a series of nonimprovements, followed by a jump in performance when all bottleneck threads finally get help. In Fig. 8, we verify this effect. The figure plots the speedup as a function of the number of threads, for different tuple costs. The threads are inserted in a balanced way, by picking the thread that executes the highest number of operators and partitioning it into two threads.

We observe that, for sufficiently high per-tuple processing costs, the speedup is a piece-wise function which jumps at certain number of threads, like 2, 4, and 8 . Each such jump point corresponds to a partitioning where all threads execute the same number of operators. This result justifies our algorithm design which inserts multiple threaded ports in one round. For low per-tuple processing costs (such as $2^{8}$ ) the speedup is not ideal, and for very low per-tuple processing costs (such as $2^{4}$ ), the performance degrades.

\subsubsection{Adaptation}

We evaluate the adaptation capability of our solution by turning on autopipelining in an application whose topology is a simple chain of Functor operators. For this experiment, we measure the throughput of the application as a function of time. The adaptation period is set to 5 seconds. We report the throughput relative to the sequential case. Fig. 9 reports these results for different per-tuple processing costs.

We observe that our algorithm intelligently achieves optimal speedup for different per-tuple costs. For instance, when the per-tuple cost is $2^{4}$, our algorithm finds out that its second optimization decision does not improve overall throughput, and thus it rolls back to the previous state. For higher per-tuple costs, such as $2^{20}$, the algorithm does not stop adding threaded ports until it reaches the unpartitionable state, that is 1 operator per thread. Comparing Figs. 8 and 9, we see that autopipelining lands on the globally optimal configuration in terms of the throughput.

The total adaptation time of the system depends on two major components: 1) the number of steps taken, and 2) the

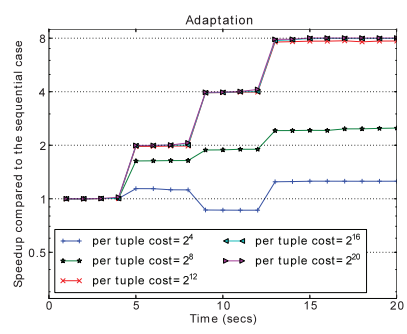

Fig. 9. Adaptation with autopipelining. 


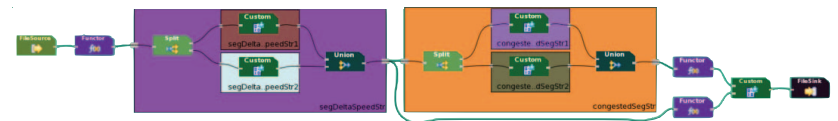

Fig. 10. LinearRoad—A vehicle toll computation app.

adaptation period. Since our algorithm helps all bottlenecked threads at each step, its behavior with respect to the number steps taken is favorable. For instance, it takes $\log _{2}(8)=3$ steps to reach eight threads in Fig. 9. For more dynamic scenarios, we can reduce the adaptation period to reduce the overall adaptation time. The only downside is that, reducing the adaptation period without decreasing the accuracy of the profiling data requires increasing the profile sampling rate, which can increase the profiling cost.

\subsection{Application Benchmarks}

The application benchmarks consist of three real-world stream processing applications with their associated workloads. These applications are named Lois, Vwap, and LinearRoad. The LinearRoad application (the smallest of the three) is depicted in Fig. 10, whereas other applications are depicted in Appendix G, which is available in the online supplemental material.

The Lois [20] data set is collected from a Scandinavian radio-telescope under construction in northwestern Europe. The goal of the Lois application is to detect cosmic ray showers by processing the live data received from the radio-telescope.

The Vwap [21] data set contains financial market data in the form of a stream of real-time bids and quotes. The goal of the Vwap application is to detect bargains and trading opportunities based on the processing of the live financial feed.

LinearRoad [22] data set contains speed, direction, and position data for vehicles traveling on road segments. The goal of the application is to compute tolls for vehicles traveling on the hypothetical "Linear Road" highway.

The breakdown of the operators constituting the applications and summaries of the application characteristics are given in Appendix $G$, which is available in the online supplemental material. It is important to note that the Lois and LinearRoad applications have few bush segments in their topology, whereas Vwap has many. The LinearRoad application makes heavy use of custom operators, whereas the other applications are composted of mostly built-in operators.

We run three versions of these programs: unoptimized, hand-optimized, and autopipelined. The hand-optimized versions are created by explicitly inserting threaded ports in the SPL code of the application. This was carried out by the application developers, independent of our work. For all cases, we measure the total execution time for the entire data set. For the autopipelined version, the adaptation period is also included as part of the total execution time.

Fig. 11 gives the results. For the Lois application, we see around $1.5 \times$ speedup compared to the unoptimized version, for Vwap we see around $3 \times$ speedup, and for LinearRoad we see $2.56 \times$ speedup. Note that these are realworld applications, where sequential portions and I/O bound pieces (sources and sinks) make it difficult to attain

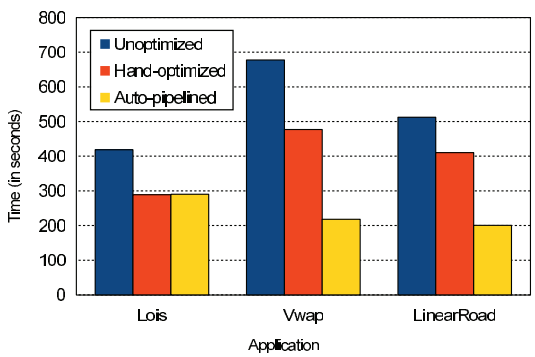

Fig. 11. Running time for Lois and Vwap.

perfect speedup. It is impressive that our autopipelining solution matches the hand-optimized performance in the case of Lois, and improves upon it by around $2 \times$ for both Vwap and LinearRoad. It is also worth noting that in the case of Lois, the programmer has statically added threaded ports based on her experience and the suggestion from a fusion optimization tool called COLA [16]. Considering that the autopipeliner takes around 20 seconds to adapt in this particular case, the throughout attained for the autopipelining solution is in fact higher than the hand-optimized case.

Overall, autopipelining provides equal or significantly better performance compared to hand optimization, at no additional cost to the application developers.

\section{Related Work}

Our work belongs to the area of autoparallelization and we survey the related topics accordingly. Coverage of related work on profiling is given in the Appendix $\mathrm{H}$, which is available in the online supplemental material.

Dynamic multithreaded concurrency platforms, such as Cilk++ [8], OpenMP [7], and $\times 10$ [12], decouple expressing a program's innate parallelism from its execution configuration. OpenMP and Cilk++ are widely used language extensions for shared memory programs, which help express parallel execution in a program at developmenttime and take advantage of it at runtime.

Kremlin [23] is an autoparallelization framework that complements OpenMP [7]. Kremlin recommends to programmers a list of regions for parallelization, which is ordered by achievable program speedup.

Cilkview [24] is a Cilk++ analyzer of program scalability in terms of number of cores. Cilkview performs systemlevel modeling of scheduling overheads and predicts program speedup. Bounds on the speedup are presented to programmers for further analysis.

Autopin [25] is an autoconfiguration framework for finding the best mapping between system cores and threads. Using profile runs, Autopin exhaustively probes all possible mappings and finds the best pinning configuration in terms of performance.

StreamIt [26] is a language for creating streaming applications and can take advantage of parallelism present in data flow graph representation of applications, including task, pipeline, and data parallelism. However, StreamIt is mostly a synchronous streaming system, where static scheduling is performed based on compile-time analysis of filters written in the StreamIt language.

Alchemist [27] is a dependence profiling technique based on postdominance analysis and is used to detect candidate 
regions for parallel execution. It is based on the observation that a procedure with few dependences with its continuation benefits more from parallelization.

Task assignment in distributed computing has been an active research problem for decades. General task assignment is intractable. In [28], several programs with special structures are considered and the optimal assignment is found by using a graph theoretic approach.

There has been extensive research in the literature on compiler support for instruction-level or fine-grained pipelined parallelism [29]. In this work, we look at coarsegrained pipelining techniques that address the problem of decomposing an application into higher level pieces that can execute in pipeline parallel.

Relevant to our study is the work in [30], which provides compiler support for coarse-grained pipelined parallelism. To automate pipelining, it selects a set of candidate filter boundaries (a middleware interface exposed by DataCutter [31]), determines the communication volume for these boundaries, and performs decomposition and code generation to minimize the execution time. To select the best filters, communication costs across each filter boundary are estimated by static program analysis and a dynamic programming algorithm is used to find the optimal decomposition.

A more detailed analysis of the differences of our work from others is given in Appendix I, which is available in the online supplemental material.

\section{Conclusion}

In this paper, we described an autopipelining solution for data stream processing applications. It automatically discovers pipeline and task parallelism opportunities in stream processing applications, and applies dynamic profiling and controlling to adjust the level of parallelism needed to achieve the best throughput. Our solution is transparent in the sense that no changes are required on the application source code. Our experimental evaluation shows that our solution is also effective, matching or exceeding the speedup that can be achieved via expert tuning. Our solution has been implemented on a commercial-grade data stream processing system. We provide directions for future work in Appendix $\mathrm{K}$, which is available in the online supplemental material.

\section{REFERENCES}

[1] A. Arasu, B. Babcock, S. Babu, M. Datar, K. Ito, R. Motwani, I. Nishizawa, U. Srivastava, D. Thomas, R. Varma, and J. Widom, "STREAM: The Stanford Stream Data Manager," IEEE Data Eng. Bull., vol. 26, no. 1, 2003.

[2] D. Abadi, Y. Ahmad, M. Balazinska, U. Çetintemel, M. Cherniack, J.-H. Hwang, W. Lindner, A. Maskey, A. Rasin, E. Ryvkina, N. Tatbul, Y. Xing, and S. Zdonik, "The Design of the Borealis Stream Processing Engine," Proc. Second Biennial Conf. Innovative Data Systems Research (CIDR), 2005.

[3] N. Jain, L. Amini, H. Andrade, R. King, Y. Park, P. Selo, and C. Venkatramani, "Design, Implementation and Evaluation of the Linear Road Benchmark on the Stream Processing Core," Proc. ACM SIGMOD Int'l Conf. Management of Data, 2006.

[4] StreamBase Systems, http://www.streambase.com, Oct. 2011.

[5] B. Gedik and H. Andrade, "A Model-Based Framework for Building Extensible, High Performance Stream Processing Middleware and Programming Language for IBM Infosphere Streams," Software: Practice and Experience, vol. 42, pp. 13631391, 2012.
[6] S4 Distributed Stream Computing Platform, http://www.s4.io/, Oct. 2011.

[7] Openmp, http://www.openmp.org, Oct. 2011.

[8] Cilk++. http://software.intel.com/en-us/articles/intel-cilk-plus / , Oct. 2011.

[9] J. Reinders, Intel Threading Building Blocks: Outfitting C++ for MultiCore Processor Parallelism. O'Reilly, 2007.

[10] B.L. Chamberlain, D. Callahan, and H.P. Zima, "Parallel Programmability and the Chapel Language," Int'l J. High Performance Computing Applications, vol. 21, pp. 291-312, 2007.

[11] G.L. Steele Jr., "Parallel Programming and Code Selection in Fortress," Proc. 11th ACM SIGPLAN Symp. Principles and Practice of Parallel Programming (PPoPP), 2006.

[12] P. Charles, C. Grothoff, V.A. Saraswat, C. Donawa, A. Kielstra, K. Ebcioglu, C. von Praun, and V. Sarkar, "X10: An Object-Oriented Approach to Non-Uniform Cluster Computing," Proc. 20th Ann. ACM SIGPLAN Conf. Object-Oriented Programming, Systems, Languages, and Applications (OOPSLA), 2005.

[13] B. Gedik, H. Andrade, K.-L. Wu, P.S. Yu, and M. Doo, "SPADE: The System S Declarative Stream Processing Engine," Proc. ACM SIGMOD Int'l Conf. Management of Data, 2008.

[14] M.M. Astrahan et al., "System R: A Relational Approach to Data Management," ACM Trans. Database Systems, vol. 1, no. 2, pp. 97137, 1976.

[15] B. Gedik, H. Andrade, and K.-L. Wu, "A Code Generation Approach to Optimizing High-Performance Distributed Data Stream Processing," Proc. 18th ACM Conf. Information and Knowledge Management (CIKM), 2009.

[16] R. Khandekar, K. Hildrum, S. Parekh, D. Rajan, J.L. Wolf, K.-L. Wu, H. Andrade, and B. Gedik, "COLA: Optimizing Stream Processing Applications via Graph Partitioning," Proc. ACM/IFIP/ USENIX 10th Int'l Conf. Middleware (Middleware), 2009.

[17] J. Giacomoni, T. Moseley, and M. Vachharajani, "FastForward for Efficient Pipeline Parallelism: A Cache-Optimized Concurrent Lock-Free Queue," Proc. 13th ACM SIGPLAN Symp. Principles and Practice of Parallel Programming (PPoPP), 2008.

[18] S.L. Graham, P.B. Kessler, and M.K. McKusick, "gprof: A Call Graph Execution Profiler (With Retrospective)," Proc. ACM SIGPLAN Conf. Programming Language Design and Implementation (PLDI), pp. 49-57, 1982.

[19] TCMalloc: Thread-Caczhing Malloc, http://goog-perftools. sourceforge.net/doc/tcmalloc.html, Aug. 2012.

[20] Lois, http://www.lois-space.net/, Oct. 2011.

[21] H. Andrade, B. Gedik, K.-L. Wu, and P.S. Yu, "Processing High Data Rate Streams in System S," J. Parallel and Distributed Computing, vol. 71, no. 2, pp. 145-156, 2011.

[22] A. Arasu, S. Babu, and J. Widom, "The CQL Continuous Query Language: Semantic Foundations and Query Execution," The VLDB J., vol. 15, no. 2, pp. 121-142, 2006.

[23] S. Garcia, D. Jeon, C.M. Louie, and M.B. Taylor, "Kremlin: Rethinking and Rebooting Gprof for the Multicore Age," Proc. 32nd ACM SIGPLAN Conf. Programming Language Design and Implementation (PLDI), 2011.

[24] Y. He, C.E. Leiserson, and W.M. Leiserson, "The Cilkview Scalability Analyzer," Proc. 22nd ACM Symp. Parallelism in Algorithms and Architectures (SPAA), 2010.

[25] T. Klug, M. Ott, J. Weidendorfer, and C. Trinitis, "Autopin: Automated Optimization of Thread-to-Core Pinning on Multicore Systems," Trans. High-Performance Embedded Architectures and Compilers, vol. 3, pp. 219-235, 2011.

[26] M.I. Gordon, W. Thies, and S. Amarasinghe, "Exploiting CoarseGrained Task Data, and Pipeline Parallelism in Stream Programs," Proc. 12th Int'l Conf. Architectural Support for Programming Languages and Operating Systems (ASPLOS), 2006.

[27] X. Zhang, A. Navabi, and S. Jagannathan, "Alchemist: A Transparent Dependence Distance Profiling Infrastructure," Proc. IEEE/ACM Seventh Ann. Int'l Symp. Code Generation and Optimization (CGO), pp. 47-58, 2009.

[28] S.H. Bokhari, Assignment Problems in Parallel and Distributed Computing. Kluwer Academic Publishing, 1987.

[29] S.M. Krishnamurthy, "A Brief Survey of Papers on Scheduling for Pipelined Processors," ACM SIGPLAN Notices, vol. 25, no. 7, pp. 97-106, 1990.

[30] W. Du, R. Ferreira, and G. Agrawal, "Compiler Support for Exploiting Coarse-Grained Pipelined Parallelism," Proc. ACM/ IEEE Conf. Supercomputing (SC), p. 8, 2003. 
[31] M.D. Beynon, T.M. Kurç, Ü.V. Çatalyürek, C. Chang, A. Sussman, and J.H. Saltz, "Distributed Processing of Very Large Data Sets with Datacutter," Parallel Computing J., vol. 27, no. 11, pp. 14571478, 2001.

[32] E. Jeřábek, “Dual Weak Pigeonhole Principle, Boolean Complexity, and Derandomization," Annals of Pure and Applied Logic, vol. 129, pp. 1-37, 2004.

[33] S. Liang and D. Viswanathan, "Comprehensive Profiling Support in the Java Virtual Machine," Proc. Fifth Conf. USENIX ObjectOriented Technologies and Systems (COOTS), 1999. pp. 229-242.

[34] Oprofile, http://oprofile.sourceforge.net/about/, Oct. 2011.

[35] J.A.M. Anderson, L.M. Berc, J. Dean, S. Ghemawat, M.R. Henzinger, S.T. Leung, R.L. Sites, M.T. Vandevoorde, C.A. Waldspurger, and W.E. Weihl, "Continuous Profiling: Where Have All the Cycles Gone?" Proc. 16th ACM Symp. Operating Systems Principles (SOSP), pp. 1-14, 1997.

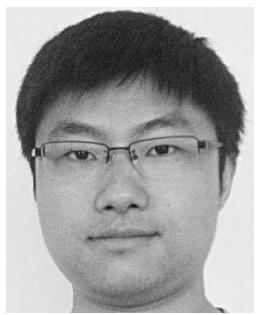

Yuzhe Tang received the BSc and MSc degrees in computer science and engineering from Fudan University, Shanghai, China, in 2006 and 2009 , respectively. $\mathrm{He}$ is currently working toward the PhD degree at the Data Intensive Distributed Systems Lab, College of Computing, Georgia Institute of Technology. At the time of this writing he was an intern at the IBM T. J. Watson Research Center working with Dr Gedik on high-performance streaming systems. His research interests include distributed systems and cloud computing, databases, system security and privacy. He has worked on HBase and Hadoop ecosystem, profiling and system optimizations, anonymity protocols and data management over DHT networks. He is a student member of the IEEE.

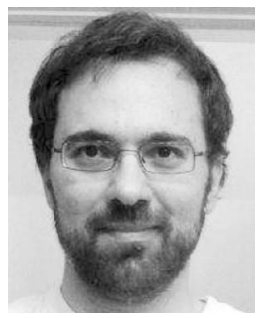

Buğra Gedik received the BS degree in computer engineering and information science from Bilkent University, Turkey, and the PhD degree in computer science from Georgia Institute of Technology. He is currently an assistant professor at the Computer Engineering Department, Bilkent University, Turkey. Prior to that he worked as a research staff member at the IBM T.J. Watson Research Center. His research interests include distributed data-intensive systems with a particular focus on stream computing. In the past, he served as the chief architect for IBM's InfoSphere Streams product. $\mathrm{He}$ is the coinventor of the SPL and the SPADE stream processing languages. He is the corecipient of the IEEE ICDCS 2003, IEEE DSN 2011, ACM DEBS 2011, and 2012 best paper awards. He served as the co-PC chair for the ACM DEBS 2009 and IEEE CollaborateCom 2007 conferences. He is an associate editor for the IEEE Transactions on Services Computing journal. He served on the program committees of numerous conferences, including IEEE ICDCS, VLDB, ACM SIGMOD, IEEE ICDE, and EDBT. He has published more than 60 peer-reviewed articles in the areas of distributed computing and data management. He has applied for more than 30 patents, most of them related to his work on streaming technologies. He was named an IBM master inventor and is the recipient of an IBM Corporate Award for his work in the System $S$ project. He is a member of the IEEE.

$\checkmark$ For more information on this or any other computing topic, please visit our Digital Library at www.computer.org/publications/dlib. 